\title{
Dissemination of Direct Healthcare Professional Communications on Medication Errors for Medicinal Products in the EU: An Explorative Study on Relevant Factors
}

\author{
Christina E. Hoeve ${ }^{1,2}\left(\mathbb{D} \cdot\right.$ Esther de Vries ${ }^{2,3} \cdot$ Peter G. M. Mol ${ }^{2,3} \cdot$ Miriam C. J. M. Sturkenboom ${ }^{4}$. \\ Sabine M. J. M. Straus ${ }^{1,2}$
}

Published online: 23 December 2020

(c) The Author(s) 2020

\begin{abstract}
Introduction When serious medication errors (ME) are identified, communication to the field may be necessary. In the EU, communication of serious safety issues, such as medication errors associated with adverse drug reactions, is done through direct healthcare professional communications (DHPCs). We aimed to identify how often DHPCs about medication errors are distributed, and we explored factors associated with these ME DHPCs.

Methods We performed a descriptive study of all centrally authorised products (CAPs) approved before 1 May 2019 in the EU. All DHPCs issued between 1 January 2001 and 1 May 2019 were reviewed for ME content. Characteristics of CAPs were collected from the website of the European Medicines Agency. A Kaplan-Meier survival analysis was performed to estimate the 5- and 10-year probability of the occurrence of a first ME DHPC. A logistic regression was performed to explore risk factors for ME DHPCs.

Results A total of 678 CAPs were included, of which 35 required an ME DHPC during the study period. The 5-year probability for a CAP to have a first ME DHPC was $2.5 \%$ (95\% CI 1.1-3.9) and the 10-year probability was 4.4\% (95\% CI 2.2-6.5). Among products with an ME DHPC, the 5-year probability of a second ME DHPC was 21.3\% (95\% CI 0.2-38.0). The risk of ME DHPCs was increased for products with multiple pharmaceutical formulations, enteral liquid or parenteral injection preparations, and products classified as nervous system agents or antineoplastic and immunomodulating agents.

Conclusions The absolute number of ME DHPCs for CAPs is low and does not give rise to immediate concern. We identified potential risk factors for ME DHPCs that should be taken into account during approval procedures or line extensions.
\end{abstract}

Electronic supplementary material The online version of this article (https://doi.org/10.1007/s40264-020-00995-4) contains supplementary material, which is available to authorized users.

Christina E. Hoeve

c.hoeve@erasmusmc.nl

1 Department of Medical Informatics, Erasmus Medical Center, Rotterdam, The Netherlands

2 Medicines Evaluation Board, Utrecht, The Netherlands

3 University Medical Center Groningen, Groningen, The Netherlands

4 Julius Global Health, University Utrecht Medical Center, Utrecht, The Netherlands

\section{Key Points}

The probability for a centrally authorised medicinal product in the EU to have a medication error DHPC within 5 and 10 years after authorisation is low $(2.5 \%$ and $4.4 \%$, respectively).

Products with multiple pharmaceutical formulations, enteral liquid or parenteral injection preparations, and products classified as nervous system agents or antineoplastic and immunomodulating agents have an increased risk of requiring a medication error DHPC. 


\section{Introduction}

Medication errors and unsafe medication practices have been identified as an important cause for avoidable harm worldwide, with an estimated global cost of US\$42 billion annually [1]. Medication errors can occur at any stage of the treatment process. Measures to minimise the risk of medication errors should be developed by pharmaceutical companies before the product reaches the market. This can be done, for example, with the use of a unique product name to limit product name confusion or clear packaging to limit look-a-like confusion [2]. The European Medicines Agency (EMA) has released guidance on risk minimisation of medication errors that can be used both pre-marketing and post-marketing [3]. Since 2012, all new products authorised in the European Union (EU) should develop a risk management plan (RMP) as part of the application procedure [4, 5]. In this RMP the Marketing Authorisation Holder (MAH) should discuss the potential for medication errors and the need for risk minimisation measures [6]. Routine risk minimisation measures may consist of, for example, clear packaging or instructions in the patient leaflet, or pack size limitation. In specific situations, regulators can request additional risk minimisation measures from pharmaceutical companies to minimise the risk of medication errors. However, not all risks for medication errors are foreseen during the development phase. A number of factors may play a role in the occurrence of medication errors in clinical practice, for example, confusion with other products on the market, confusion between pharmaceutical formulations, or shortages of products, resulting in the use of replacement drugs. These errors are only identified when the product is on the market.

When serious medication errors are identified (i.e. medication errors with serious adverse drug reactions), communication of these risks to the field may be necessary. In the EU, Direct Healthcare Professional Communications (DHPCs) provide communication of important safety issues to the field [7]. A DHPC is a letter containing information on "new information about a previously known or unknown risk of a medicine which has or could have an impact on a medicine's risk-benefit balance and its condition of use" [7]. In addition, it should contain recommendations to the healthcare professional (HCP) on how to deal with the safety concern, which could include recommendations on how to avoid medication errors [7]. DHPCs for centrally authorised products (CAPs) are discussed by the Pharmacovigilance Risk Assessment Committee (PRAC) and/or the Committee for Medicinal Products for Human Use (CHMP). A DHPC will be prepared by the MAH after receiving a request from the regulatory agency. The DHPC is prepared through cooperation between the MAH and regulators, and is usually disseminated by MAHs, after both parties have agreed on the content. When medication errors are identified post-marketing, a DHPC may be disseminated; however, if a potential risk for medication errors is identified before or at the time of approval, a DHPC may be disseminated at the time of launch as a risk minimisation measure. However, if a new strength or formulation of an already marketed product is introduced that may lead to confusion and an ME, a DHPC at the time of this introduction might be necessary.

There is currently only limited knowledge on the factors influencing the occurrence of DHPCs for medication errors. This study aims to identify the number of medication errors that required communication via DHPCs. We also explored factors associated with the dissemination of DHPCs specifically regarding the risk of medication errors.

\section{Methods}

We designed a descriptive study of all CAPs. The study period to identify DHPCs ranged from 1 January 2001 to 30 June 2019. All CAPs authorised before 1 May 2019 were included unless they were not originator CAPs (innovative products with a new active substance).

\subsection{Data Collection and Covariates}

Further information on the CAPs was extracted from publicly available information on the website of the European Medicines Agency (https://www.ema.europa.com). The website provides structured information on all authorised CAPs, including active substance, Anatomical Therapeutic Chemical (ATC) code, authorisation status (authorised, refused, suspended, withdrawn), legal basis (generic, biosimilar, conditional approval, exceptional circumstances, accelerated assessment, orphan medicine), status of additional monitoring (yes/no) and date of authorisation. The legal basis was obtained from the European Public Assessment Report (EPAR) or the application form for the application procedure. ${ }^{1}$ If no information regarding legal basis of the product authorisation could be retrieved, these products were excluded from further analysis. Date of withdrawal and reason for withdrawal were obtained from the public statement on withdrawal of the products, which is available on the EMA website. Pharmaceutical form(s) and number of pharmaceutical forms were collected for each product

\footnotetext{
${ }^{1}$ Possible legal basis for a medicinal product in the EU: 8.3 Full or full-mixed application, 8.3 (multiple), 10a Well established use application, 10b Fixed dose combination, 10c Informed consent application, 10(1) Generic medicinal product application, 10(3) Hybrid medicinal product application, 10(4) Similar biologic product application.
} 
from the EPAR-All Authorised presentations, which is available on the EMA website. Products were classified based on administration route (see supplementary table in the electronic supplementary material [ESM] for categorisation). For products with an RMP at the time of application, information on the safety specification was collected, including if medication errors were included as a safety concern (important identified risk, important potential risk, missing information or not classified), and whether additional risk minimisation measures for medication errors were applicable. RMPs were obtained from the initial application dossier. From all included DHPCs, information was collected on the type of medication error and the source of information that triggered the DHPC. Further, DHPCs were categorised by response type (reactive and preventive). DHPCs were reactive when they were disseminated post-marketing (e.g. in response to an occurring safety issue) and preventive when they were disseminated at product launch.

\subsection{Exclusion Criteria}

CAPs that were not originator products were excluded from the analysis, as they are in general expected to follow the originator in the dissemination of DHPCs. Inclusion of these products would therefore dilute the potential associations between product characteristics and DHPC dissemination.

\subsection{Outcome}

Our main outcome was the occurrence of a DHPC that was issued between 1 January 2001 and 30 June 2019 for medication errors (ME DHPC), which was defined as a DHPC describing unintended failure in the drug treatment process that led to, or had the potential to lead to, harm to the patient. ${ }^{2}$ Follow-up time was defined as time from authorisation to first ME DHPC, withdrawal or end of study period, whichever came first.

\subsection{Data Analysis}

Descriptive statistics were used for the characteristics of the CAPs. Kaplan-Meier survival analysis was performed to estimate the 5- and 10-year probability of the occurrence of a first DHPC for medication errors with and without stratification for ME category. For the Kaplan-Meier survival analysis, follow-up time was defined as the time from authorisation to DHPC, and the denominator was all CAPs authorised after 2000. A sensitivity analysis investigated the 5-year

\footnotetext{
${ }^{2}$ Refer to the European Medicines Agency's definition of medication errors as described in the Good practice guide on recording, coding, reporting and assessment of medication errors.
}

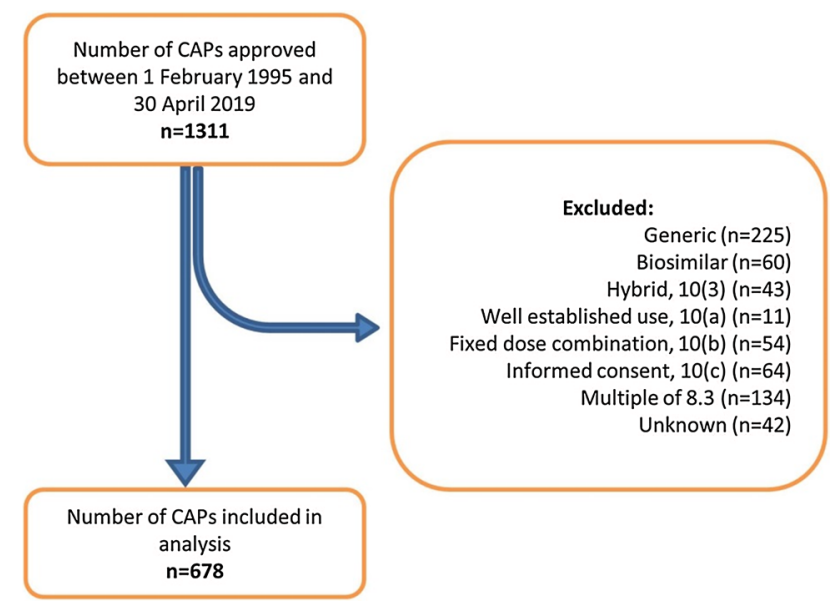

Fig. 1 Exclusion of non-originator products authorised in the EU during the study period

probability for a first ME DHPC before and after 2012. For products with an ME DHPC, the 5- and 10-year probability of the occurrence of a second ME DHPC was calculated. A median time to ME DHPC was calculated based only on products for which ME DHPCs were distributed. Odds ratios were calculated for different exposure categories using logistic regression. All data were processed in Microsoft Excel and analysed using R version 3.6.1 (packages used: glm, survival, dplyr, tidyr).

\section{Results}

A total of 1311 CAPs were authorised in the European Economic Area (EEA) before 1 May 2019. Of the 1311 products, 633 applications were excluded as they did not concern applications for new originator products (Fig. 1). The 678 originator CAPs authorised in the EEA were included for the analysis. In total, 319 DHPCs were issued for these CAPs during the study period, of which 35 DHPCs (11.0\%, 95\% CI 7.8-14.9) concerned medication errors (ME DHPCs). The rate of ME DHPCs issued per year ranged between zero and five and the proportion of ME DHPCs over all DHPCs fluctuated between $0.0 \%$ and $23.5 \%$ per year during the entire study period (Fig. 2). The 35 ME DHPCs were issued for 28 CAPs, which corresponds to $4.1 \%$ of 678 CAPs authorised before 1 May 2019 and included in this study. Among all CAPs, the 5-year probability for a medical substance to have a first ME DHPCs was 2.5\% (95\% CI 1.1-3.9) and the 10 -year probability was $4.4 \%$ (95\% CI 2.2-6.5). A sensitivity analysis to evaluate the 5-year probability for a CAP to have a first ME DHPC before and after 2012 showed that CAPs authorised before 2012 had a $2.4 \%$ (95\% CI 
Fig. 2 Yearly number of Direct Healthcare Professional Communications for Centrally Authorised Products communicating on medication errors

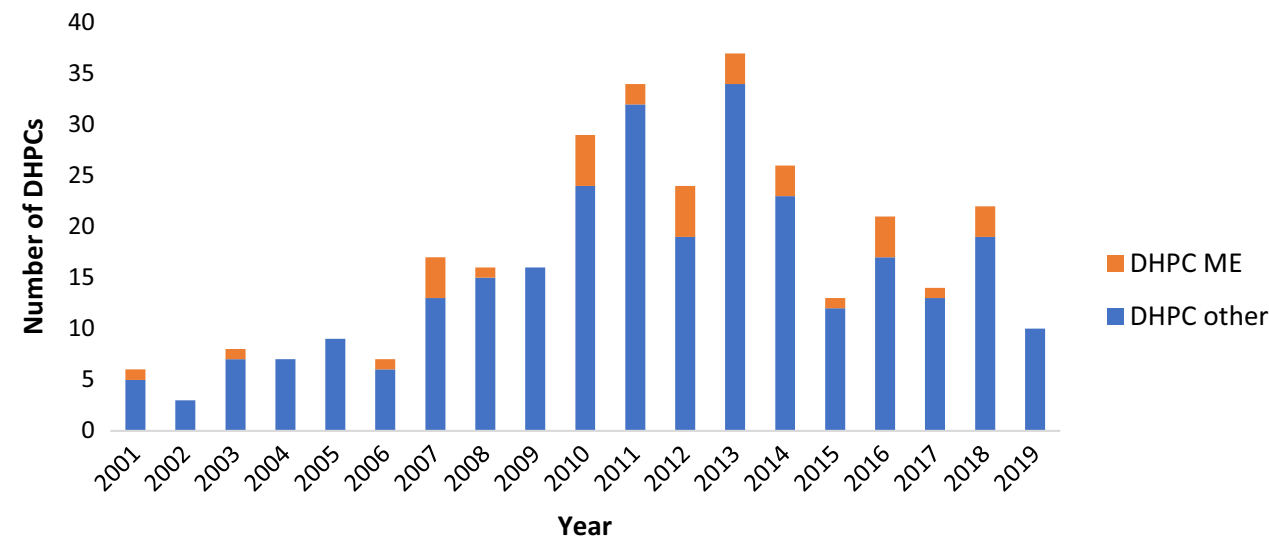

Table 1 Types of medication errors discussed in Direct Healthcare Professional Communications

\begin{tabular}{|c|c|c|c|c|}
\hline Error category & Data source & $N(\%)$ & $\begin{array}{l}5 \text {-year probability of } \\
\text { ME DHPC }(95 \% \text { CI })^{b}\end{array}$ & $\begin{array}{l}\text { 10-year probability of } \\
\text { ME DHPC }(95 \% \mathrm{CI})^{\mathrm{b}}\end{array}$ \\
\hline Confusion due to changes to the product & $\begin{array}{l}\text { PM safety reports [3] } \\
\text { Preventive [6] } \\
\text { PM complaints [1] }\end{array}$ & $10(28.6)$ & $0.67(0.00-0.89)$ & $0.83(0.00-0.97)$ \\
\hline Confusion due to unclear information/packaging & $\begin{array}{l}\text { PM safety reports [2] } \\
\text { Preventive [2] }\end{array}$ & $4(11.4)$ & 0.00 (NA-NA) & 1.00 (NA-NA) \\
\hline Confusion with other formulations/strengths/products & $\begin{array}{l}\text { PM safety reports [2] } \\
\text { Preventive [4] }\end{array}$ & $6(17.1)$ & $0.60(0.00-0.86)$ & $0.80(0.00-0.97)$ \\
\hline Inappropriate use of product with quality issue & PM complaints [4] & $4(11.4)$ & $0.33(0.00-0.70)$ & $0.33(0.00-0.70)$ \\
\hline Non-adherence to instructions & $\begin{array}{l}\text { PM safety reports }{ }^{\mathrm{a}}[6] \\
\text { Clinical trial data }{ }^{\mathrm{a}}[1] \\
\text { Observational study [1] } \\
\text { PM complaints [1] }\end{array}$ & $8(22.9)$ & $0.43(0.00-0.70)$ & $0.86(0.12-0.98)$ \\
\hline Other & $\begin{array}{l}\text { PM safety reports [2] } \\
\text { Unclear [1] }\end{array}$ & $3(8.6)$ & $0.33(0.00-0.70)$ & 1.00 (NA-NA) \\
\hline
\end{tabular}

The 5-year and 10-year probability for error categories were calculated including only products for which an ME DHPC had been disseminated CAPs centrally authorised products, DHPC Direct Healthcare Professional Communication, ME medication error, NA not applicable, PM postmarketing

${ }^{\text {a }}$ One DHPC was based on both PM safety reports and clinical trial data and was counted twice

${ }^{\mathrm{b}}$ For the Kaplan-Meier survival analysis, only the CAPs authorised after 2000 were included. As a result, seven CAPs were excluded from this analysis

0.6-4.1) probability for a CAP to have a first ME DHPC after 5 years. For CAPs authorised after 2012, this was $3.1 \%$ (95\% CI 0.1-5.9). Among the 28 CAPs for which ME DHPCs were issued, one CAP was withdrawn before the end of the study period for commercial reasons. For products with an ME DHPC, the median time from approval to DHPC was 5.2 years (range 0.7-19.2). The ME DHPC issued earliest after approval (0.7 years) was for tenecteplase (Metalyse ${ }^{\circledR}$, Boehringer Ingelheim), concerning the risk of dosing errors if the product was not used correctly. The ME DHPC that was sent out the longest after marketing approval (19.2 years) was for cetrorelix (Cetrotide ${ }^{\circledR}$, Merck) and was based on spontaneous reports describing a risk of administration of non-sterile product due to incorrect use of a newly introduced syringe.

\subsection{Second/Third DHPC}

For six CAPs, a second ME DHPC was issued between 0.9 and 8.3 years after the first DHPC. Among products with an ME DHPC, the 5-year probability of a second ME DHPC was $21.3 \%$ (95\% CI 0.2-38.0). In two cases, the second DHPC concerned the same safety issue. For one CAP (levetiracetam [Keppra ${ }^{\circledR}$, UCB Pharma]) a third DHPC was issued 6.3 years after the second DHPC. All three DHPCs for this product concerned different medication error issues. 


\subsection{Medication Error Categories}

The 35 ME DHPCs issued were most frequently related to confusion due to changes to the product $(29 \%)$, non-adherence to instructions (23\%), and confusion with other formulations, strengths or products from the same marketing authorisation (17\%) (Table 1). Confusion due to changes to the product was most frequently related to confusion at the level of HCPs $(n=8 / 10)$, and less frequently at the level of the patient $(n=5 / 10)$. For example, with the introduction of a new oral levetiracetam dosing system (from a syringe with milligrams to a syringe with millilitres), it was important to inform both HCPs and patients about the potential confusion between the old and the new system (supplementary table, see ESM). Examples of ME DHPCs specifically targeting HCPs were for radium-223 dichloride (Xofigo ${ }^{\circledR}$, Bayer AG) and moroctocog alfa (ReFacto $\mathrm{AF}^{\circledR}$, Pfizer). The reference standard was revised for these products, which introduced a risk of incorrect dosing (supplementary table, see ESM). Confusion with other formulations, strengths or products was more often related to the level of patients $(n=5 / 6)$ than HCPs $(n=4 / 6)$. Confusion due to unclear product information or packaging occurred more often at the level of HCPs $(n=4 / 4)$ than patients $(n=1 / 4)$. Among products with an ME DHPC, errors due to confusion as a result of changes to the product, unclear information/packaging or confusion with other formulations/strengths/products were communicated fastest with a 5-year probability of 50\% (Table 1). ME DHPCs were categorised as reactive or preventive DHPCs based on source of information. The majority of ME DHPCs were issued post-marketing and were triggered by spontaneous reports, product complaints, clinical trials or observational studies $(n=24)$. Twelve ME DHPCs were issued at approval to prevent potential anticipated MEs (Table 1). Preventive ME DHPCs were disseminated to communicate the potential risk of confusion as a result of changes to the products, unclear information/packaging or confusion with other formulations/strengths/products. The most common errors described in preventive ME DHPCs were the risk of incorrect dosing due to the introduction of new batches with a new reference standard $(n=3)$, risk of confusion between two available formulations or strengths $(n=3)$ and risk of dosing due to availability of dosing information based on the salt form and information based on the active substance $(n=2)$ (supplementary table, see ESM).

\subsection{Drug Class}

The majority of CAPs for which ME DHPCs were sent out concerned antineoplastic and immunomodulating agents ( $n=10,34.5 \%)$, followed by medicines targeting the nervous system $(n=5,17.2 \%)$ and medicines targeting blood and blood-forming organs $(n=4,13.8 \%)$ (Table 2). We observed increased odds of an ME DHPC for antineoplastic and immunomodulating agents (OR 7.99; 95\% CI 1.39-49.77) and for nervous systems medication (OR 8.31; 95\% CI 1.66-38.57) compared with anti-infectives for systemic use (Table 2). A high variety of types of errors and their consequences was observed within drug classes (see supplementary table in the ESM). An example of a risk for an immunomodulating agent was confusion between the dosing schedule of tacrolimus (Advagraf ${ }^{\circledR}$, Astellas Pharma Europe) and tacrolimus (Prograft ${ }^{\circledR}$, Astellas Pharma), both indicated for prophylaxis of transplant rejection in adult kidney or liver allograft recipients. These products contain the same active substance, but have a different dosing scheme. Confusion between these schedules may lead to acute transplant rejection or toxicity due to overdose. All risks for other products are described in the supplementary table (see ESM).

\subsection{Approval Procedure}

CAPs authorised with exceptional circumstances, with conditional approval or as an orphan drug did not have an increased risk of an ME DHPC (Table 2). None of the products in our dataset were approved through an accelerated assessment procedure.

\subsection{Risk Management Plan}

Among the 678 included CAPs, $72.0 \%$ had an RMP at the time of authorisation (Table 2). For CAPs with an RMP at the time of marketing authorisation, CAPs with ME safety concerns in the RMP had a higher chance of requiring an ME DHPC during post-marketing than CAPs without ME safety concerns in the RMP, although this was not statistically significant (OR 1.97; 95\% CI 0.44-8.72). CAPs without RMP had higher odds for an ME DHPC than CAPs with an RMP without ME safety concerns (OR 3.53; 95\% CI 1.22-10.22) (Table 2). CAPs with an RMP at registration had a 5-year probability of a first ME DHPC of $2.4 \%$ (95\% CI 0.8-3.9), whereas CAPs without an RMP at registration had a 5-year probability of a first ME DHPC of 2.8\% (95\% CI 0.0-5.9). For all four products with ME safety concerns in the RMP and an ME DHPC, the safety concern was the same as the ME discussed in the DHPC (Table 3). In addition, two of the four products already had educational material implemented to minimise the risk of the medication errors addressed in the DHPC.

\subsection{Pharmaceutical Formulation}

The risk of an ME DHPC was significantly increased for products with two (OR 16.74; 95\% CI 1.80-155.57) or three different pharmaceutical formulations (OR 222.65; 95\% CI 
Table 2 Univariate and multivariate analysis of factors associated with disseminated ME DHPCs

\begin{tabular}{|c|c|c|c|c|c|c|}
\hline Characteristic & $\begin{array}{l}\text { Total } N=678 \\
(\% \text { of all } \\
\text { CAPs })\end{array}$ & $\begin{array}{l}\text { CAPs with ME } \\
\text { DHPC (\% of } \\
\text { total) } \\
N=29(4.3 \%)\end{array}$ & $\begin{array}{l}\text { Univariate } \\
\text { odds ratio }\end{array}$ & $95 \%$ confidence interval & $\begin{array}{l}\text { Multivari- } \\
\text { ate odds } \\
\text { ratio }\end{array}$ & $95 \%$ confidence interval \\
\hline \multicolumn{7}{|l|}{ Drug class } \\
\hline $\begin{array}{l}\text { A-alimentary tract and } \\
\text { metabolism }\end{array}$ & 84 & $2(2.4)$ & 1.07 & $0.17-6.51$ & 3.29 & $0.42-25.58$ \\
\hline $\begin{array}{l}\text { B-blood and blood-form- } \\
\text { ing organs }\end{array}$ & 55 & $4(7.3)$ & 3.42 & $0.74-15.84$ & 5.49 & $0.90-33.31$ \\
\hline $\mathrm{C}$-cardiovascular system & 28 & $0(0.0)$ & 0.00 & 0.00 & 0.00 & 0.00 \\
\hline D_dermatologicals & 10 & $0(0.0)$ & 0.00 & 0.00 & 0.00 & 0.00 \\
\hline $\begin{array}{l}\mathrm{G} \text { - } \text { genitourinary system } \\
\text { and sex hormones }\end{array}$ & 23 & $0(0.0)$ & 0.00 & 0.00 & 0.00 & 0.00 \\
\hline $\begin{array}{l}\mathrm{H}-\text { systemic hormonal } \\
\text { preparations, excluding sex } \\
\text { hormones and insulins }\end{array}$ & 11 & $1(9.1)$ & 4.37 & $0.42-45.91$ & 6.19 & $0.47-80.91$ \\
\hline $\begin{array}{l}\text { J—anti-infectives for sys- } \\
\text { temic use }\end{array}$ & 134 & $3(2.2)$ & 1.00 & Reference & 1.00 & Reference \\
\hline $\begin{array}{l}\mathrm{L} \text {-antineoplastic and } \\
\text { immunomodulating agents* }\end{array}$ & 179 & $10(5.6)$ & 2.58 & $0.70-9.58$ & 7.99 & $1.66-38.57$ \\
\hline $\mathrm{M}$-musculoskeletal system & 20 & $1(5.0)$ & 2.30 & $0.23-23.24$ & 1.46 & $0.09-23.15$ \\
\hline $\mathrm{N}$-nervous system & 53 & $5(9.4)$ & 4.55 & $1.05-19.76$ & 8.31 & 1.39-49.77 \\
\hline $\begin{array}{l}\mathrm{P} \text {-antiparasitic products, } \\
\text { insecticides and repellents }\end{array}$ & 1 & $0(0.0)$ & 0.00 & 0.00 & 0.00 & 0.00 \\
\hline $\mathrm{R}$-respiratory system & 19 & $0(0.0)$ & 0.00 & 0.00 & 0.00 & 0.00 \\
\hline $\mathrm{S}$-sensory organs & 22 & $1(4.5)$ & 2.08 & $0.21-20.94$ & 6.13 & $0.32-117.20$ \\
\hline $\mathrm{V}$-various & 36 & $1(2.8)$ & 1.25 & $0.13-12.37$ & 0.95 & $0.07-13.67$ \\
\hline NYA—not yet assigned & 3 & $0(0.0)$ & 0.00 & 0.00 & 0.00 & 0.00 \\
\hline Orphan drug (yes) & $117(17.3)$ & $1(0.9)$ & 0.17 & $0.02-1.27$ & 0.12 & $0.01-1.27$ \\
\hline Conditional approval (yes) & $20(2.9)$ & $1(5.0)$ & 1.23 & $0.16-9.53$ & 5.86 & $0.47-72.81$ \\
\hline $\begin{array}{l}\text { Exceptional circumstances } \\
\text { (yes) }\end{array}$ & $27(4.0)$ & $0(0.0)$ & 0.00 & 0.00 & 0.00 & 0.00 \\
\hline \multicolumn{7}{|l|}{ ME as safety concern in EU RMP } \\
\hline Yes & $111(16.4)$ & $4(3.6)$ & 1.98 & $0.57-6.88$ & 1.97 & $0.44-8.72$ \\
\hline No & $377(55.6)$ & $7(1.9)$ & 1.00 & Reference & 1.00 & Reference \\
\hline No RMP & $190(28.0)$ & $17(8.9)$ & 5.19 & $2.12-12.76$ & 3.53 & $1.22-10.22$ \\
\hline \multicolumn{7}{|l|}{ Type of administration } \\
\hline Topical & $30(4.4)$ & $0(0.0)$ & 0.00 & 0.00 & 0.00 & 0.00 \\
\hline $\begin{array}{l}\text { Parenteral (through injec- } \\
\text { tions) }\end{array}$ & $291(42.9)$ & $13(4.5)$ & 10.24 & $1.33-78.89$ & 10.29 & $1.23-86.38$ \\
\hline Parenteral (other) & $18(2.7)$ & $0(0.0)$ & 0.00 & 0.00 & 0.00 & 0.00 \\
\hline Enteral (solid) & $220(32.4)$ & $1(0.5)$ & 1.00 & Reference & 1.00 & Reference \\
\hline Enteral (liquid) & $15(2.2)$ & $1(6.7)$ & 15.64 & $0.93-263.48$ & 53.09 & 2.50-1126.01 \\
\hline Other & $16(2.4)$ & $2(12.5)$ & 31.29 & $2.67-366.38$ & 56.77 & 2.90-1112.33 \\
\hline Missing & $3(0.4)$ & $1(33.3)$ & 109.50 & $4.92-2435.06$ & 69.73 & $2.46-1972.78$ \\
\hline Two formulations & $73(1.0)$ & $6(8.2)$ & 19.61 & $2.32-165.80$ & 16.74 & 1.80-155.57 \\
\hline Three formulations & $11(1.6)$ & $4(36.4)$ & 125.14 & 12.34-1269.50 & 222.65 & $15.00-3305.16$ \\
\hline Four formulations & $1(0.1)$ & $0(0.0)$ & 0.00 & 0.00 & 0.00 & 0.00 \\
\hline
\end{tabular}

The column total contains percentages of all CAPs per category; the column CAPs with ME DHPCs contains percentages of the total CAPs with and without ME DHPCs in the corresponding group

Odds ratios presented bold are statistically significant

CAPs centrally authorised products, $D H P C$ Direct Healthcare Professional Communication, EU European Union, $M E$ medication error, $R M P$ risk management plan 


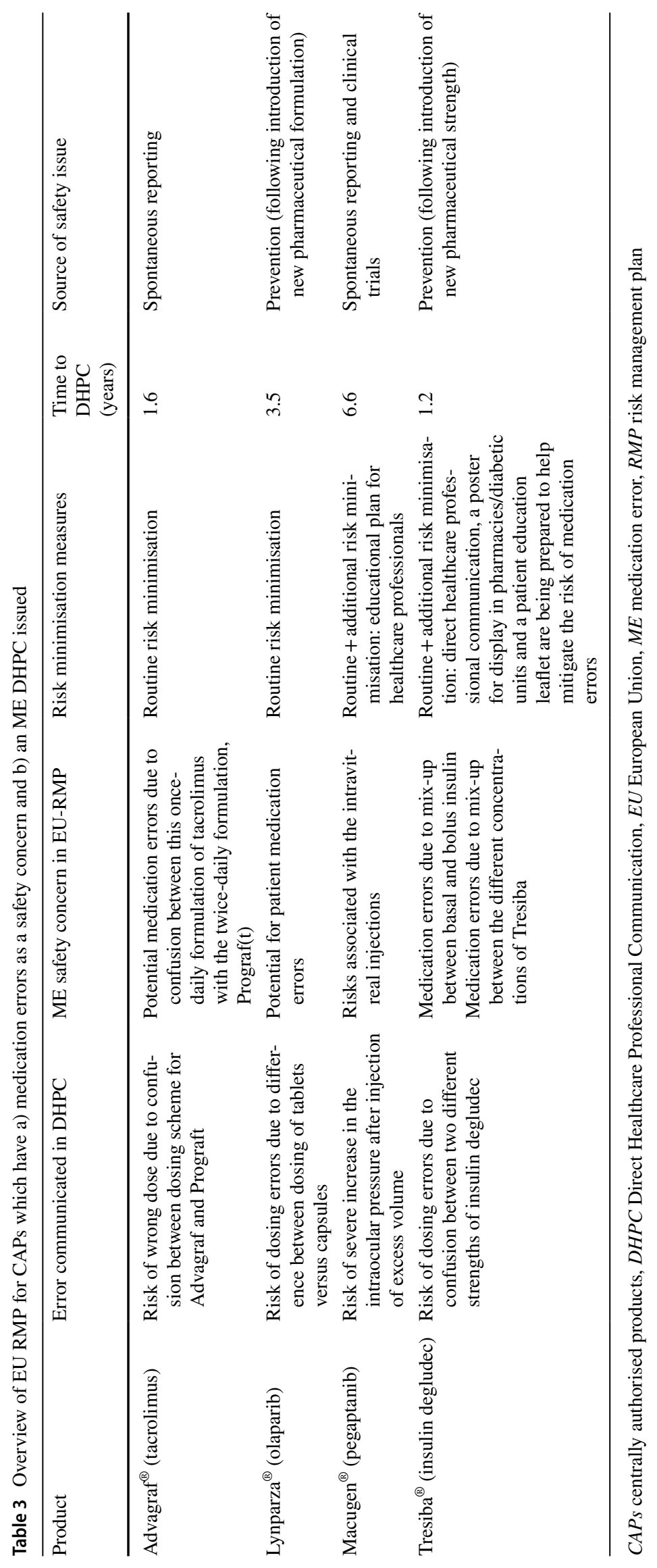


15.00-3305.16) compared with products with only a solid enteral formulation (Table 2). In total, ten ME DHPCs in our dataset were for products with multiple formulations. Review of the ME risks showed that in four of the ten cases, the ME risk was directly related to the existence of multiple formulations. An example is the confusion between tablets and suspension formulations of posaconazole (Noxafil ${ }^{\circledR}$, Merck Sharp and Dohme). Posaconazole is prescribed for the treatment of infections. The DHPC informed HCPs that the oral suspension and tablets are not interchangeable, and direct substitution without recalculation of the dose could lead to underdose or overdose. The type of pharmaceutical formulation also influenced the odds of an ME DHPC. The chance of an ME DHPC significantly increased (OR 10.29; 95\% CI 01.23-86.38) for products with a parenteral injection and (OR 53.09; 95\% CI 2.50-1126.01) for products with an enteral liquid formulation.

\section{Discussion}

CAPs authorised before 1 May 2019 had a 5-year probability of $2.5 \%$ (95\% CI 1.1-3.9) and 10-year probability of $4.4 \%$ (95\% CI 2.2-6.5) to have a first ME DHPC. The sensitivity analysis showed no difference in 5-year probability before and after 2012. However, the numbers are small and limit firm conclusions. Spontaneous reports were most often the source for ME DHPCs. Mol et al. observed a rate of $6.5 \%$ ME DHPCs among DHPCs issued in the Netherlands [8]. In that study, national and decentralised products were also included and therefore cannot be used as a direct comparison. While ME-related harm is a large concern in clinical practice, our study shows that only in a limited number of cases a DHPC is required. This suggests that the prevention of ME remains challenging and requires involvement and efforts of all stakeholders.

We demonstrated that the type and number of pharmaceutical formulations as well as the class of drug are important risk factors for a product to have an ME DHPC after marketing. Products with parenteral injections, enteral liquids or multiple formulations had a significantly higher risk of requiring an ME DHPC than products with only an enteral solid formulation. Parenteral injections and enteral liquids required more preparation steps like measuring liquids, dissolving and diluting and the administration is often more complex. The increased risk of medication errors with use of parenteral injections is also supported by Valentin et al., who observed that more severely ill patients had a higher risk of medication errors, which can be explained by the fact that these patients require more complex care and use more parenteral medication [9]. Half of the medication error safety issues for enteral liquid products concerned wrong administration in children. Oral liquids in children have been listed before as a risk factor for medication errors [10-12]. The doses are often dependent on the size and weight of the child and need to be measured individually. In addition, measurements through dosing syringes may cause confusion to caregivers or healthcare professionals [10-12]. Moreover, our data show that there were multiple issues with syringes due to introduction of new measuring scales, confusion between milligrams and millilitres or fading of the measuring scales. Having different formulations for a product increased the risk of an ME DHPC. At licensure, a clear benefit-risk assessment should be made to evaluate whether additional formulations really benefit the patient. An example that shows how the benefit-risk balance may deviate per formulation is dextropropoxyphene (although not related to medication errors). During the dextropropoxyphene referral in 2006-2008, there was an evaluation of the benefit risk of dextropropoxyphene in the light of potential abuse resulting in fatal overdose. During this referral it was decided that the product was suspended for all formulations except IV formulations (which are only administered in hospitals), since these had no abuse potential.

Anti-neoplastic and immunomodulating agents and nervous system agents received significantly more ME DHPCs compared with anti-infectives for systemic use. In the EEA, medication errors are frequently reported for nervous system products and antineoplastic products [13]. Antineoplastic agents were mostly parenteral injections (70\%) and $20 \%$ of antineoplastic agents had two formulations marketed.

All five nervous system products that required an ME DHPC had an enteral liquid formulation marketed and four of the five products had multiple formulations marketed. It could be hypothesised that errors with this type of product have more severe consequences and therefore more often require a DHPC, since DHPCs are only disseminated if the ME would lead to severe adverse events. This is supported by the fact that medication errors for these products are often reported to EudraVigilance, where only medication errors that resulted in adverse events have to be reported [13]. However, to confirm this, other data is required such as medication errors that did not need a DHPC, information on near misses and medication errors without adverse events.

Type of errors for antineoplastic agents and nervous system products were of varying nature and no particular pattern was observed for drug classes.

\subsection{Other Observations}

In total, 11 products authorised with an RMP required an ME DHPC. Interestingly, we found that for all four CAPs with medication error safety concerns in the RMP and an ME DHPC, the safety concern was the same as included in the DHPC. On the one hand, this shows that medication errors can be predicted. The majority of these DHPCs (3/4) 
concerned introduction of a new formulation or strength that could lead to confusion with already marketed products. Therefore, these risks were relatively easy to anticipate. However, it also shows that the measures implemented at authorisation were not sufficient to minimise the risk of medication errors enough, even though additional risk minimisation measures were in place for these risks in two of the four CAPs. This raises questions about the effectiveness of these measures. Furthermore, for seven CAPs the risk was not foreseen, even though there was an RMP in place. The type of error was much more varied for these seven CAPs and was often related to confusion due to changes to the product or due to unclear information/packaging. Since these changes to the product occurred post-marketing, unsurprisingly these risks were not foreseen during development of the RMP before marketing. Unclear product information or packaging, however, is a factor that could be evaluated and foreseen before marketing through human factor testing as recommended in the Good practice guide on risk minimisation and prevention of medication errors [14].

\subsubsection{Time to DHPC}

The time to ME DHPC was highly variable. Errors that were related to confusion around the product (e.g. unclear product information) were identified more rapidly than errors related to quality issues or errors related to non-adherence (an example of a quality issue is fading of measurement lines on a syringe, which may lead to dosing errors). A reason could be that medication error risks due to quality issues can be detected at any time of the product life cycle since the quality issue can occur at any time during the product's lifetime. Also, issues related to non-adherence may be introduced later in the product life cycle when HCPs assume that they are experienced with the product. Unclear product information, on the other hand, might be detected faster since it introduces a risk immediately at the start of the marketing period, which is therefore more likely to be detected early in the product life cycle.

\subsubsection{Repeat DHPCs for One Product}

There were six CAPs for which more than one new medication error issue was identified. For bivalirudin (Angiox ${ }^{\circledR}$, The Medicines Company UK), an ME DHPC was distributed twice for the same issue. This raises additional questions on the effectiveness of the communication and whether other additional measures might have been more useful for this product (e.g. educational material, stickers on packaging, etc.). No studies were identified in the medical literature evaluating the effectiveness of the DHPCs for bivalirudin and the product was withdrawn for commercial reasons in 2018 .

\section{Limitations}

We used a dataset of DHPCs that were distributed in the Netherlands regarding centrally authorised products. This means that the wording in other countries may be slightly different, but the topics are the same. Sending a DHPC is the responsibility of the national medicines agency. A minor proportion could have been missing in case a CAP was not marketed in the Netherlands at the time of DHPC distribution, or if the issue was considered not relevant in the Netherlands.

DHPCs are only one measure to minimise the risk of medication errors, other actions such as changes to the Summaries of Product Characteristics (SmPCs) are not included in this overview.

DHPCs agreed at marketing approval may be sent over a year after the date of marketing approval, as there may be a delay in the time of marketing of the product in different countries. Time to DHPC may therefore vary slightly between countries.

Since data on DHPCs was not systematically collected before 2001, we excluded CAPs authorised before 2001 from the survival analysis to avoid immortal time bias. Although generic products are expected to follow the originator in the development of their risk minimisation measures, there are situations possible where a medication error risk is specific for a generic product. Since we have only included originators in this study, some cases might be missing.

The small number of DHPCs addressing ME limited some of the analyses and interpretation. Although some factors were associated with increased or reduced risks of ME DHPCs, this should be interpreted cautiously.

Evaluation of the potential adverse events following ME was beyond the scope of this study, therefore we cannot comment on the severity of medication errors that were described in the DHPCs.

\section{Conclusion}

The absolute number of ME DHPCs for CAPs is low and does not give rise to immediate concern. Products with multiple pharmaceutical formulations, enteral liquid or parenteral injection preparations, and products classified as nervous system agents or antineoplastic and immunomodulating agents are at higher risk of an ME DHPC. These factors are relevant to take into account during approval procedures or line-extension procedures in the context of medication errors. Once a product has a DHPC, the risk of a repeat DHPC is high, therefore, more effective communication may be needed. 


\section{Declarations}

Funding No sources of funding were used in the preparation of this study.

Conflict of Interest CH, EdV, PM, SS have no conflicts of interest directly relevant to this study. Miriam Sturkenboom receives salary for the conduct of a post-authorisation safety study from Novartis through the PHARMO Institute.

Ethics Approval No ethics approval was required for this study.

Consent to Participate Not applicable.

Consent for Publication Not applicable.

Availability of Data and Material The data used in this study were derived from the website of the European Medicines Agency, a public domain resource, which can be accessed via http://www.ema.europa.eu.

Code Availability All data were processed in Microsoft Excel and analysed using R version 3.6.1 (packages used: glm, survival, dplyr, tidyr).

Author Contributions CEH: conceptualisation, data curation, formal analysis, methodology, validation, visualisation, writing — review and editing. EV: conceptualisation, data curation, writing-review and editing. PM: methodology, writing-review. MCJMS: conceptualisation, methodology, supervision. SMJMS: conceptualisation, methodology, supervision. All authors read and approved the final version.

Open Access This article is licensed under a Creative Commons Attribution-NonCommercial 4.0 International License, which permits any non-commercial use, sharing, adaptation, distribution and reproduction in any medium or format, as long as you give appropriate credit to the original author(s) and the source, provide a link to the Creative Commons licence, and indicate if changes were made. The images or other third party material in this article are included in the article's Creative Commons licence, unless indicated otherwise in a credit line to the material. If material is not included in the article's Creative Commons licence and your intended use is not permitted by statutory regulation or exceeds the permitted use, you will need to obtain permission directly from the copyright holder. To view a copy of this licence, visit http://creativecommons.org/licenses/by-nc/4.0/.

\section{References}

1. World Health Organisation. Medication without harm: global patient safety challenge. Geneva: World Health Organisation; 2017.

2. Ostini R, Roughead EE, Kirkpatrick CM, Monteith GR, Tett SE. Quality use of medicines-medication safety issues in naming; look-alike, sound-alike medicine names. Int J Pharm Pract. 2012;20(6):349-57.

3. Goedecke T, Ord K, Newbould V, Brosch S, Arlett P. Medication errors: new EU good practice guide on risk minimisation and error prevention. Drug Saf. 2016;39(6):491-500.
4. Union European. Directive 2010/84/EU of the European Parliament and of the Council of 15 December 2010 amending as regards pharmacovigilance, Directive 2001/83/EC on the Community code relating to medicinal products for human use. Off $\mathrm{J}$ Eur Union. 2010;L348(53):74-99. Found on: https://eur-lex.europ a.eu/LexUriServ/LexUriServ.do?uri=OJ:L:2010:348:0074:0099: EN:PDF.

5. European Union. Regulation (EU) No $1235 / 2010$ of the European Parliament and of the Council of 15 December 2010 amending, as regards pharmacovigilance of medicinal products for human use, Regulation (EC) No 726/2004 laying down Community procedures for the authorisation. Off J Eur Union. 2010;348(726). Found on: https://eur-lex.europa.eu/LexUriServ/LexUriServ.do? uri=OJ:L:2010:348:0001:0016:EN:PDF.

6. European Medicines Agency and Heads of Medicines Agencies. Guideline on good pharmacovigilance practices (GVP) Module V-Risk management systems (Rev 2) (EMA/838713/2011 Rev 2). 2017. Found on: https://www.ema.europa.eu/en/documents/ scientific-guideline/guideline-good-pharmacovigilance-practicesmodule-v-risk-management-systems-rev-2_en.pdf.

7. European Medicines Agency and Heads of Medicines Agencies. Guideline on good pharmacovigilance practices (GVP) Module XV—Safety communication (Rev 1) EMA/118465/2012. 2017. Found on: https://www.ema.europa.eu/en/documents/scientific -guideline/guideline-good-pharmacovigilance-practices-modul e-Xv-safety-communication-rev-1_en.pdf.

8. Mol PGM, Straus SMJM, Piening S, de Vries JTN, de Graeff PA, Haaijer-Ruskamp FM. A decade of safety-related regulatory action in the netherlands. A retrospective analysis of direct healthcare professional communications from 1999 to 2009. Drug Saf. 2010;33(6):463-74.

9. Valentin A, Capuzzo M, Guidet B, Moreno R, Metnitz B, Bauer $\mathrm{P}$, et al. Errors in administration of parenteral drugs in intensive care units: multinational prospective study. BMJ. 2009;338:b814.

10. Yin HS, Parker RM, Sanders LM, Dreyer BP, Mendelsohn AL, Bailey S, et al. Liquid medication errors and dosing tools: a randomized controlled experiment. Pediatrics. 2016;138(4):e20160357.

11. Jones AN, Miller JL, Neely S, Ibach BW, Hagemann TM, Golding $\mathrm{CL}$, et al. Prevalence of unrounded medication doses and associated factors among hospitalized pediatric patients. J Pediatr Pharmacol Ther. 2017;22(4):286-92.

12. Lovegrove MC, Sapiano MRP, Paul IM, Yin HS, Wilkins TL, Budnitz DS. Primary care provider perceptions and practices regarding dosing units for oral liquid medications. Acad Pediatr. 2018;18(4):405-8.

13. Newbould V, Le Meur S, Goedecke T, Kurz X. Medication errors: a characterisation of spontaneously reported cases in EudraVigilance. Drug Saf. 2017;40(12):1241-8.

14. Pharmacovigilance Risk Assessment Committee (PRAC). Good practice guide on risk minimisation and prevention of medication errors. 2015. Found on: https://www.ema.europa.eu/en/docum ents/regulatory-procedural-guideline/good-practice-guide-riskminimisation-prevention-medication-errors_en.pdf. 